\title{
Kinetics of humoral immune response over 17 months of COVID-19 pandemic in a large cohort of healthcare workers in Spain: The ProHEpic-19 study
}

\section{Concepción Violán ( $\nabla$ cviolanf.mn.ics@gencat.cat)}

Fundació Institut Universitari per a la Recerca a I'Atenció Primària de Salut Jordi Gol i Gurina (IDIAPJGol)

\section{Pere Torán}

Fundació Institut Universitari per a la Recerca a l'Atenció Primària de Salut Jordi Gol i Gurina (IDIAPJGol)

Bibiana Quirant

Germans Trias i Pujol Research Institute (IGTP)

\section{Noemi Lamonja-Vicente}

Fundació Institut Universitari per a la Recerca a l'Atenció Primària de Salut Jordi Gol i Gurina (IDIAPJGol)

\section{Lucía A Carrasco-Ribelles}

Fundació Institut Universitari per a la Recerca a l'Atenció Primària de Salut Jordi Gol i Gurina (IDIAPJGol)

\section{Carla Chacón}

Fundació Institut Universitari per a la Recerca a l'Atenció Primària de Salut Jordi Gol i Gurina (IDIAPJGol)

\section{Josep $M^{a}$ Manresa-Dominguez}

Fundació Institut Universitari per a la Recerca a l'Atenció Primària de Salut Jordi Gol i Gurina (IDIAPJGol)

\section{Francesc Ramos-Roure}

Institut Català de Salut

\section{Rosalia Dacosta-Aguayo}

Fundació Institut Universitari per a la Recerca a l'Atenció Primària de Salut Jordi Gol i Gurina (IDIAPJGol)

\section{Cristina Palacios}

Institut Català de Salut

\section{Albert Roso-Llorach}

Fundació Institut Universitari per a la Recerca a l'Atenció Primària de Salut Jordi Gol i Gurina (IDIAPJGol)

Aleix Pujol

AIDS Research Institute Irsicaixa

Dan Ouchi

AIDS Research Institute Irsicaixa

\section{Mónica Monteagudo}

Institut Català de Salut

\section{Pilar Montero}

Fundació Institut Universitari per a la Recerca a l'Atenció Primària de Salut Jordi Gol i Gurina (IDIAPJGol)

\section{Rosa Garcia-Sierra}

Fundació Institut Universitari per a la Recerca a l'Atenció Primària de Salut Jordi Gol i Gurina (IDIAPJGol)

\section{Fernándo Arméstar}

Hospital Universitari Germans Trias i Pujol

\section{Maria Doladé}

Hospital Universitari Germans Trias i Pujol

\section{Nuria Prat}

Institut Català de Salut 
Josep Ma Bonet

Institut Català de Salut

Bonaventura Clotet

AIDS Research Institute Irsicaixa

Ignacio Blanco

Laboratori clinic Metropolitana Nord (LCMN). Hospital Universitari Germans Trias i Pujol

Julia G Prado

AIDS Research Institute Irsicaixa

Eva Marái Martínez-Cáceres

Germans Trias i Pujol Research Institute (IGTP)

\section{Research Article}

Keywords: SARS-CoV-2, COVID-19, Antibodies, IgG, IgM, Seroprevalence, Kinetics, Humoral immunity, Clinical spectrum, Health Care Workers, Cohort, non-linear mixed models

Posted Date: February 23rd, 2022

DOl: https://doi.org/10.21203/rs.3.rs-1363425/v1

License: (c) (i) This work is licensed under a Creative Commons Attribution 4.0 International License. Read Full License 


\section{Abstract}

\section{Background}

Understanding the immune response to the SARS-CoV-2 virus is critical for efficient monitoring and defence strategies. The ProHEpic-19 cohort provides a fine-grained description of the kinetics of antibodies after SARS-CoV-2 infection with an exceptional resolution over 17 months.

\section{Methods}

We established a cohort of 769 health professionals including healthy and infected with SARS-CoV-2 in northern Barcelona to determine the kinetics of the IgM against the nucleocapsid $(\mathrm{N})$ and the IgG against the $\mathrm{N}$ and spike (S) of SARS-CoV-2. We used non-linear mixed models to investigate the kinetics of IgG and IgM measured at nine time points over 17 months from the diagnosis. The model included factors of time, gender, and disease severity (asymptomatic, mild-moderate, severecritical) to assess their effects and their interactions.

Findings

: 474 of the 769 participants (61.6\%) became infected with SARS-CoV-2. Significant effects of gender and disease severity were found for the levels of all three antibodies. Median $\operatorname{IgM}(N)$ levels were already below the positivity threshold in patients with asymptomatic and mild-moderate disease at day 270 after the diagnosis, while $\lg \mathrm{G}(\mathrm{N}$ and $\mathrm{S})$ levels remained positive at least until days 450 and 270, respectively. Kinetic modelling showed a general rise in both $\lg M(N)$ and $\lg G(N)$ levels up to day 30 , followed by a decay with a rate depending on disease severity. $\lg G(S)$ levels remained relatively constant from day 15 over time.

Interpretation

: $\operatorname{lgM}(\mathrm{N})$ and $\lg \mathrm{G}(\mathrm{N}, \mathrm{S}) \mathrm{SARS}-\mathrm{CoV}-2$ antibodies showed a heterogeneous kinetics over the 13 months. Only the IgG(S) showed a stable increase, and the levels and the kinetics of antibodies varied according to disease severity. The kinetics of IgM and IgG observed over a year also varied by clinical spectrum can be very useful for public health policies around vaccination criteria in adult population.

Funding: Regional Ministry of Health of the Generalitat de Catalunya (Call COVID19-PoC SLT16_04; NCT04885478)

\section{Research In Context}

\section{Evidence before this study}

Understanding the immune response to the SARS-CoV-2 virus is critical for efficient monitoring and defence strategies. We searched PubMed for articles published from database inception up to August 31, 2021, PubMed, BioRxiv, MedRxiv, and Research Square using search terms "COVID-19", "SARS-CoV-2", "Antibodies, IgG, IgM, Seroprevalence," "Kinetics," "Disease spectrum," "Systematic Review." Existing evidence concerning seroconversion and the kinetics of seroprevalence identified heterogeneity in the kinetic profiles of various antibodies, which can explain disease severity and outcome. Healthcare workers are typically considered to be at a higher risk; however, there a handful of longitudinal studies show significant variability. The most detailed of these studies included over 500 participants with four antibody assessments over seven months, and it reported a sustained increase of antibodies with neutralizing capacity.

\section{Added value of this study}

The ProHEpic-19 cohort provides a fine-grained description of the kinetics of antibodies after SARS-CoV-2 infection with an exceptional resolution (eight antibody assessments) and duration (over 17 months). The nonlinear mixed-effects model used in our study is better suited to capture these details than the linear models used in previous studies. Finally, in contrast with 
most studies, our study employs standardized measures in assessing SARS-CoV-2 antibodies according to WHO's recommendation, which may help resolve the issue of significant heterogeneity in the previous findings.

Our findings confirm the rapid rise and subsequent decay of IgM and IgG against the nucleocapsid protein of the virus, as well as a sustained increase of IgG against the spike protein of the virus. Moreover, we identified distinct kinetic profiles of the antibodies depending on the gender of the participant and the disease severity.

\section{Implications of all the available evidence}

The meticulous analysis of the rich ProHEpic-19 cohort according to standards and best practices provides well-grounded support for several previous findings and allows for the stratification of participants, thus informing immunity monitoring strategies and (re)vaccination policies.

\section{Background}

Infection with the SARS-CoV-2 can be detected by measuring the level of virus-specific antibodies, reflecting an immune response against a recent or previous infection [1]. Several studies described the rapid increase (Igs) of various immunoglobulin isotypes ( $\operatorname{lgA}, \operatorname{lgM}, \lg G$ ) against the epitopes of the spike (S) glycoprotein as well as to the nucleocapsid (N) protein in SARS-CoV-2 infection [2-5].

COVID-19, the disease caused by the SARS-CoV-2, has a broad clinical spectrum, including various forms of clinical presentation ranging between asymptomatic infections and critical illness. Likewise, the antibody response to the SARS-CoV2 infection is also heterogeneous [6]. Few longitudinal studies have performed serological follow-up across the clinical spectrum, and with a limited study duration between 80 to 270 days [2-4]. An early study reported a rapid rise and subsequent fall of antibodies, which stabilized at later time points, indicating that immunity against SARS-CoV-2 may last for at least four months after infection [7]. Two later studies suggested this protection for up to at least six months [8]' [2], while other recent reports claim that it persists for at least a year [9]' [10]. However, knowledge on the kinetics and the immune response to SARS-CoV-2 infection is still limited, and larger and more detailed longitudinal studies are needed to define the half-life of antibodies against SARS-CoV-2.

Of the few studies investigating the kinetics of the antibodies only one have studied IgM antibody [10]. None of these studies have more than five measurements [11]. In addition, all have made either linear or LOESS regression models, which do not allow to obtain a model that represents the non-linearity and heterogeneity of the antibody response, indicated by previous kinetics studies. The use of non-linear models over linear or LOESS models would allow to obtain a more realistic representation of the antibody kinetics that can better explain the behaviour of the antibodies.

The WHO recommends that a generic population-based serological testing should be carried out using enzyme linked immunosorbent assays (ELISA) or immunofluorescence assays (IFA) with standardized reagents [12] Using reagents in compliance with WHO's international standards for anti-SARS-CoV-2 antibodies allows to evaluate vaccine efficacy and federate and compare epidemiological and immunological surveillance studies. At the beginning of the pandemic, antibody levels were determined using qualitative assays; while using internationally standardized enzyme immunoassays for the quantitative detection of specific lgG antibodies is more recent [13]. Studies with more sample points and more accurate statistics analysis provide a better assessment of the disease burden and transmission to better inform public health efforts against COVID-19.[14] [9]

The aim of this study is to describe the kinetics of $\lg M(N)$ and $\lg G(N, S)$ antibodies against SARS-CoV-2 and assess their relationship with the clinical spectrum and other factors. Our findings provide a detailed picture of the immune response against the virus in a population which plays the most crucial role in fighting for the pandemic. Our results can guide public health policies to develop more efficient strategies for monitoring, treatment, and prevention of COVID-19. 


\section{Methods}

\section{Study design and ethics}

ProHEpiC-19 is a prospective, longitudinal study, involving two groups of health professionals (healthy and infected) in the Northern Metropolitan Area of Barcelona (Spain). The ethics committees of the Foundation University Institute for Primary Health Care Research Jordi Gol i Gurina (IDIAPJGol) (ref. 20/067) and The Germans Trias i Pujol Research Institute(IGTP) (ref.COV20/00660 (PI-20-205)) approved the study protocol, which has been published on ClinicalTrials.gov (NCT04885478). All participants recruited in the study were fully informed about the ProHEpiC-19 protocol and signed informed consent to participate. They consented to use their collected data for research and agreed to the applicable regulations, privacy policies, and terms of use. Participant data has been anonymised according to a numerical coding system based on order and stored in a database securely. The database will be maintained for a period of 15 years after the completion of the study.

No participants or members of the public were directly involved in the design or analysis of the reported data. The funders of the study had no role in study design, data collection, data analysis, data interpretation, or writing of the report. The corresponding author (CV) had full access to all data, while LACR, ARLL, DO, JMMD, and AP; had access to the raw data.

\section{Participant recruitment, follow-up}

Health professionals (physicians, nurses, nursing assistant, researchers, and other essential workers) in direct contact with patients during the first, second or third wave of COVID-19 were recruited between 3 March 2020 and 22 March 2021. Inclusion criteria were agreeing to participate and confirming their availability for the follow-up sessions $7,15,30,60,90,180$, 270, and 360, and 450 days and, 18, 24,30, and 36 months after their first visit (baseline). The first follow-up was on 5 May 2020 and the last on 11 November 2021. Participants with less than two visits were considered to be a drop-out. At baseline, participants were allocated to their groups (infected or uninfected) based on RT-PCR, as well as $\lg M(N)$ and $\lg G(N)$ antibody tests: If any of the two tests was positive, the participant was considered infected. The analyses reported in this work included only participants with SARS-CoV-2 antibodies.

Participants completed several clinical questionnaires and were examined for COVID-19-specific symptoms during the baseline visit, when the RT-PCR test with nasal and oropharyngeal swab and the antibody tests were performed. The RT-PCR test was repeated at the second visit, 7 days after the baseline visit. The antibody tests were repeated at 15, 30, 60, 90, 180, 270,360 , and 450 days following the baseline visit. The analysis of $\operatorname{lgG}(\mathrm{S})$ levels stopped as participants were vaccinated, while the $\lg \mathrm{M}(\mathrm{N})$ and $\lg \mathrm{G}(\mathrm{N})$ continued. Infected participants were divided into three different disease severity subgroups according to their symptomology: 1) asymptomatic: no symptoms; 2) mild-moderate: people with one or more clinical symptoms characteristic of COVID-19 and who did not require hospital admission; 3) severe-critical: patients who required hospital admission. The characteristics of the whole ProHEpiC-19 cohort, including the healthy group, at baseline are described in Table 1 
Table 1

Demographics and PCR testing for the participants according to their disease severity. Categoric variables are described as $\mathrm{N}$ (\%), and numeric variables as median (IQR) [min, max].

\begin{tabular}{|c|c|c|c|c|c|}
\hline & $\begin{array}{l}\text { Negative at baseline and } \\
\text { during follow-up } \\
\mathrm{N}=295 \text { (38.4) }\end{array}$ & $\begin{array}{l}\text { Asymptomatic } \\
\mathrm{N}=73 \text { (9.5) }\end{array}$ & $\begin{array}{l}\begin{array}{l}\text { Mild- } \\
\text { moderate } \\
\text { illness }\end{array} \\
\begin{array}{l}\mathrm{N}=363 \\
(47.2)\end{array}\end{array}$ & $\begin{array}{l}\text { Severe-critical } \\
\text { illness } \\
\mathrm{N}=38 \text { (4.9) }\end{array}$ & $\begin{array}{l}\text { Total } \\
\mathrm{N}=769\end{array}$ \\
\hline Age (years) & $46(38.5-56)[19-66]$ & $\begin{array}{l}45(31-52) \\
{[19-66]}\end{array}$ & $\begin{array}{l}45(35-53) \\
{[18-66]}\end{array}$ & $\begin{array}{l}56.5(50-61) \\
{[30-66]}\end{array}$ & $\begin{array}{l}46(36-54) \\
{[18-66]}\end{array}$ \\
\hline \multicolumn{6}{|l|}{$\begin{array}{l}\text { Gender assigned at } \\
\text { birth }\end{array}$} \\
\hline Female & $238(80.7)$ & $48(65.7)$ & $274(75.5)$ & $18(47.4)$ & $578(75.2)$ \\
\hline Male & $57(19.3)$ & $25(34.3)$ & $89(24.5)$ & $20(52.6)$ & $191(24.8)$ \\
\hline \multicolumn{6}{|l|}{ Profession } \\
\hline Doctor & $112(38.0)$ & $9(12.3)$ & $76(20.9)$ & $13(34.2)$ & $210(27.3)$ \\
\hline Nurse & 95 (32.2) & $19(26.0)$ & $96(26.4)$ & $10(26.3)$ & $220(28.6)$ \\
\hline Nurse assistant & $13(4.41)$ & $9(12.3)$ & $34(9.37)$ & $4(10.5)$ & $60(7.80)$ \\
\hline Others & $75(25.4)$ & $36(49.3)$ & $157(43.3)$ & $11(28.9)$ & $279(36.3)$ \\
\hline \multicolumn{6}{|c|}{ Highest educational level attained } \\
\hline $\begin{array}{l}\text { Higher level } \\
\text { vocational school }\end{array}$ & $34(11.8)$ & $6(8.57)$ & $32(9.22)$ & $1(2.78)$ & $73(9.84)$ \\
\hline University & $27(9.34)$ & $29(41.4)$ & $94(27.1)$ & $10(27.8)$ & $160(21.6)$ \\
\hline Others & $228(78.9)$ & $35(50.0)$ & $221(63.7)$ & $25(69.4)$ & $509(68.6)$ \\
\hline NA & 6 & 3 & 16 & 2 & 27 \\
\hline \multicolumn{6}{|l|}{ Marital status } \\
\hline Single & $44(15.3)$ & $16(25.0)$ & $63(18.2)$ & $3(8.33)$ & $126(17.1)$ \\
\hline Married/cohabitation & $196(68.1)$ & $42(65.6)$ & $255(73.5)$ & $29(80.6)$ & $522(71.0)$ \\
\hline Divorced & 43 (14.9) & $3(4.69)$ & $22(6.34)$ & $3(8.33)$ & $71(9.66)$ \\
\hline Widow & $5(1.74)$ & $3(4.69)$ & $7(2.02)$ & $1(2.78)$ & $16(2.18)$ \\
\hline NA & 7 & 9 & 16 & 2 & 34 \\
\hline \multicolumn{6}{|l|}{ Nationality } \\
\hline Spain & $270(95.4)$ & $57(90.5)$ & $291(87.7)$ & $35(100)$ & $653(91.6)$ \\
\hline European Union & $1(0.35)$ & $0(0.00)$ & $1(0.30)$ & $0(0.00)$ & $2(0.28)$ \\
\hline South America & $7(2.47)$ & $1(1.59)$ & $23(6.93)$ & $0(0.00)$ & $31(4.35)$ \\
\hline Others & $5(1.77)$ & $5(7.94)$ & $17(5.12)$ & $0(0.00)$ & 27 (3.79) \\
\hline NA & 12 & 10 & 31 & 3 & 59 \\
\hline
\end{tabular}




\begin{tabular}{|c|c|c|c|c|c|}
\hline & $\begin{array}{l}\text { Negative at baseline and } \\
\text { during follow-up } \\
\mathrm{N}=295 \text { (38.4) }\end{array}$ & $\begin{array}{l}\text { Asymptomatic } \\
\mathrm{N}=73 \text { (9.5) }\end{array}$ & $\begin{array}{l}\text { Mild- } \\
\text { moderate } \\
\text { illness } \\
\mathrm{N}=363 \\
(47.2)\end{array}$ & $\begin{array}{l}\text { Severe-critical } \\
\text { illness } \\
\mathrm{N}=38(4.9)\end{array}$ & $\begin{array}{l}\text { Total } \\
\mathrm{N}=769\end{array}$ \\
\hline $\begin{array}{l}\text { Number of symptoms } \\
\text { at baseline }\end{array}$ & - & $0(0)[0-0]$ & $\begin{array}{l}7(4-9)[1- \\
17]\end{array}$ & $\begin{array}{l}9(8-11)[2- \\
17]\end{array}$ & $\begin{array}{l}6(2-9)[0-17] \\
(*)\end{array}$ \\
\hline \multirow[t]{2}{*}{ Days of follow-up } & \multirow[t]{2}{*}{$\begin{array}{l}424(269.5-481)[13- \\
545]\end{array}$} & $\begin{array}{l}302(189- \\
365)\end{array}$ & $\begin{array}{l}350(271- \\
434.5)\end{array}$ & $\begin{array}{l}438.5(357.8- \\
475.5)\end{array}$ & $\begin{array}{l}363(269- \\
464)\end{array}$ \\
\hline & & [14-527] & [13-537] & [156-505] & [13-545] \\
\hline \multirow[t]{2}{*}{$\begin{array}{l}\text { Days since first } \\
\text { positive diagnosis test }\end{array}$} & \multirow[t]{2}{*}{-} & $\begin{array}{l}302(220.5- \\
374.5)\end{array}$ & \multirow[t]{2}{*}{$\begin{array}{l}365(287- \\
429)[28- \\
617]\end{array}$} & \multirow[t]{2}{*}{$\begin{array}{l}492(341.8- \\
540.8)[28- \\
582]\end{array}$} & \multirow[t]{2}{*}{$\begin{array}{l}370(286- \\
510)()^{*}[14- \\
617]\end{array}$} \\
\hline & & [14-602] & & & \\
\hline $\begin{array}{l}\geq 1 \text { positive PCR } \\
\text { during follow-up }\end{array}$ & $0(0)$ & $20(27.4)$ & $118(32.5)$ & $2(5.3)$ & $204(26.5)$ \\
\hline NA: not available & & & & & \\
\hline
\end{tabular}

Table 2

A

\begin{tabular}{|c|c|c|c|c|c|c|c|c|c|c|c|c|c|c|c|c|}
\hline \multicolumn{3}{|c|}{ Antibody } & \multicolumn{14}{|c|}{ Timepoint } \\
\hline \multirow{3}{*}{$\begin{array}{l}\lg M \\
(\mathbb{N})\end{array}$} & \multirow{3}{*}{$\begin{array}{l}\lg G \\
(N)\end{array}$} & \multirow{3}{*}{$\begin{array}{l}\text { IgG } \\
\text { (S) }\end{array}$} & \multirow{2}{*}{\multicolumn{2}{|c|}{$\begin{array}{l}0 \text { days } \\
N=142 \\
\left(75.9 \%^{*}\right)\end{array}$}} & \multirow{2}{*}{\multicolumn{2}{|c|}{$\begin{array}{l}15 \text { days } \\
\begin{array}{l}N=184 \\
(88.5 \% *)\end{array}\end{array}$}} & \multirow{2}{*}{\multicolumn{2}{|c|}{$\begin{array}{l}30 \text { days } \\
N=243 \\
(85.0 \% *)\end{array}$}} & \multirow{2}{*}{\multicolumn{2}{|c|}{$\begin{array}{l}60 \text { days } \\
N=298 \\
(84.2 \% *)\end{array}$}} & \multirow{2}{*}{\multicolumn{2}{|c|}{$\begin{array}{l}90 \text { days } \\
N=316 \\
\left(74.9 \%^{*}\right)\end{array}$}} & \multirow{2}{*}{\multicolumn{2}{|c|}{$\begin{array}{l}180 \text { days } \\
N=229 \\
(59.6 \% *)\end{array}$}} & \multirow{2}{*}{\multicolumn{2}{|c|}{$\begin{array}{l}270 \text { days } \\
N=93 \\
(26.6 \% *)\end{array}$}} \\
\hline & & & & & & & & & & & & & & & & \\
\hline & & & $\mathbf{N}$ & $\%$ & $\mathbf{N}$ & $\%$ & $\mathbf{N}$ & $\%$ & $\mathbf{N}$ & $\%$ & $\mathbf{N}$ & $\%$ & $\mathbf{N}$ & $\%$ & $\mathbf{N}$ & $\%$ \\
\hline $\mathrm{Neg}$ & Neg & Neg & 55 & 38.7 & 23 & 12.5 & 20 & 8.2 & 31 & 10.4 & 29 & 9.2 & 25 & 10.9 & 11 & 11.8 \\
\hline Neg & $\mathrm{Neg}$ & Pos & 17 & 12.0 & 28 & 15.2 & 23 & 9.5 & 17 & 5.7 & 14 & 4.4 & 25 & 10.9 & 17 & 18.3 \\
\hline Neg & Pos & $\mathrm{Neg}$ & 12 & 8.5 & 7 & 3.8 & 11 & 4.5 & 10 & 3.4 & 13 & 4.1 & 8 & 3.5 & 1 & 1.1 \\
\hline Neg & Pos & Pos & 7 & 4.9 & 15 & 8.2 & 23 & 9.5 & 35 & 11.7 & 40 & 12.7 & 42 & 18.3 & 21 & 22.6 \\
\hline Pos & $\mathrm{Neg}$ & $\mathrm{Neg}$ & 5 & 3.5 & 2 & 1.1 & 1 & 0.4 & 0 & 0 & 0 & 0 & 0 & 0 & 0 & 0 \\
\hline Pos & $\mathrm{Neg}$ & Pos & 5 & 3.5 & 2 & 1.1 & 1 & 0.4 & 0 & 0 & 3 & 1.0 & 2 & 0.9 & 0 & 0 \\
\hline Pos & Pos & $\mathrm{Neg}$ & 5 & 3.5 & 6 & 3.3 & 12 & 4.9 & 18 & 6.0 & 20 & 6.3 & 15 & 6.6 & 5 & 5.4 \\
\hline Pos & Pos & Pos & 36 & 25.4 & 101 & 54.9 & 152 & 62.6 & 187 & 62.8 & 197 & 62.3 & 112 & 48.9 & 38 & 40.9 \\
\hline
\end{tabular}

\section{SARS-CoV-2 detection and quantification of IgM and IgG}

RT-PCR was used as the primary diagnostic test. RNA for RT-PCR testing was extracted from fresh samples using the STARMag 2019-nCoV kit by means of a liquid-dispensing robot. RNA detection was performed using the Allplex SARS-CoV-2 assay, a multiplex RT-PCR assay able to detect four SARS-CoV-2 target genes in a single tube. For the antibody tests, we conducted a pre-validation study with six different commercially available and IVD-CE-approved ELISA tests and selected an anti-SARS-CoV-2 IgG(N) and IgM(N) enzyme immunoassay (ELISA) kits based on their performance. Infected participants were also tested for and measured the level of antibodies against the spike (S) subunit of SARS-CoV-2 by means of the DECOV1901 ELISA kit, which allows quantitative measurement of IgG. Please, see study protocol NCT04885478 (also attached as supplementary material) to more details. 
We used two data sources: A) A database has been created for the ProHEpiC-19 cohort. The database is stored, curated, and validated in a centralized data warehouse at the Catalan Health Institute, and it is suitable to manage the demographic, social, and clinical data of professionals; (2) results of the SARS-COV-2 antibody tests; (3) results of the RT-PCR test. B) We also implemented a Teleform-based data collection specific for the study, which includes the unique numeric patient identifier and the results of the serological and immunological tests. The unique numeric patient identifier allows to link the two data sources.

\section{Sample size}

Sample size and power estimations can be found in the supplementary protocol. The one-way ANOVA of the total collected sample of 478 infected participants (72,367, and 39 from the three subgroups) was estimated to achieve a $100 \%$ power to detect differences among the means versus the alternative of equal means using an $\mathrm{F}$ test with a 0.05 significance level and an effect size of $\eta^{2}=0.06$.

\section{Statistical analysis}

Missing values were found only in in the following sociodemographic variables: education (3.5\%), marital status (4.6\%), and nationality (7.5\%).

The evolution of antibody test was considered both as a categorical (i.e. positive/negative result) and as a continuous scalar variable. Descriptive analyses were performed to characterise the immune response at each timepoints with the occurrence and frequency for categorical variables and median and inter-quartile range for numeric variables with non-normal distributions confirmed. The antibody levels were analysed with respect to time, disease severity, and gender. We stratified antibody levels by days since diagnosis, describing them by boxplots and comparing the timepoints using a Kruskal-Wallis test, followed by Holm-adjusted Dunn's test. Then, at each timepoint, differences were assessed according to disease severity (Kruskal-Wallis followed by Holm-adjusted Dunn's test) and (Mann-Whitney U test). For a more detailed description of the antibody kinetics, we also fitted locally estimated scatterplot smoothing models (LOESS) and calculated their $95 \%$ confidence intervals (Cls). Finally, non-linear mixed effects models (NLME) were also fitted to obtain the curves characterising the antibody kinetics. In these models, each parameter was assumed to have a fixed and a random effect Both LOESS and NLME models were first fitted on data from all patients and then on data stratified by disease severity and gender. Model diagnostics were performed based on residual analysis, and goodness-of-fit was checked with Akaike and Bayesian Information Criteria (AIC and BIC). For these all analyses except LOESS, time from diagnosis was discretised into time bins so that tests performed between the designated time points were considered as performed at the earlier time point. Therefore, tests performed in the first 14 days since diagnosis are treated as "Day 0" and tests performed between day 450 and 615 (i.e. the last day observed, see Table 1) are treated as "Day 450". For the complete list and description of the time bins, see the supplementary material and Table $\mathrm{S} 1$.

All tests were two-sided, and statistical probability of $p<0.05$ was considered significant. All analyses were performed using $\mathrm{R}$ version 4.0.4. See supplementary material for more information about the statistical methods.

\section{Results}

\section{Participant characteristics}

A total of 860 participants were recruited, of whom 769 were eligible; 443 (57.6\%) tested positive at baseline, and 31 (4\%) had their first positive SARS-CoV-2 antibody test during follow-up (Figure S1, Table 1). Women had more often asymptomatic or mild disease, while men tended to have more severe disease ( $p$-value $=0.001$ ). Differences in the prevalence of specific clinical symptoms according to disease severity and gender are shown in Table S2.

\section{Seroprevalence}


Table 2A presents the frequency of each possible combination of antibody test results at each time-point. At baseline, more than one third of the participants with available data (38.7\%) tested negative for all antibodies, but this proportion decreased over time. From day 15 to day 180 of infection, more than $45 \%$ of participants tested positive for all antibodies. However, this proportion also decreased from day 180 as the number of participants with positive $\operatorname{lgM}(\mathrm{N})$ values fell. By day $270,11.8 \%$ of the participants were negative for all antibody tests, while $88.2 \%$ of the participants were positive for at least $\lg G(N)$ or $\lg G(S)$. As Table 2B shows, $68.2 \%$ of participants still had $\lg \mathrm{G}(\mathrm{N})$ values over the positivity threshold at day 360 .

\section{Levels of SARS-CoV-2 antibodies stratified by gender and disease severity}

We found a statistically significant difference in all antibody levels across clinical conditions $(p<0.001$ both overall and pairwise comparisons). There was also a significant difference in antibody levels between men and women for all immunoglobulins: $\lg M(N)(p=0.01), \lg G(N)(p<0.001)$, and $\lg G(S)(p=0.006)$.

Figure 1 shows the differences in antibody levels between the different time-points. Pairwise comparisons show that both $\lg M(N)$ and $\lg G(N)$ levels from day 270 are no longer significantly different from those on day 0 (Figs. 1A-B). Both IgM(N) and $\lg \mathrm{G}(\mathrm{N})$ levels present a rise until and fall from day 30 (Fig. 1A-B), while $\lg \mathrm{G}(\mathrm{S})$ levels remain relatively constant following the first rise at day 15 (Fig. 1C). All antibody levels of patients with severe-critical disease were the highest up to day 180 $(\operatorname{IgM}(\mathrm{N}))$, day $450(\operatorname{lgG}(\mathrm{N})$ and day $270(\operatorname{IgG}(\mathrm{S}))($ Fig. 1D-F). Resulting p-values are shown in the figure. Median IgM(N) levels were below the positivity threshold in people with asymptomatic and mild-moderate diseases after day 270 from diagnosis (Fig. 1D). However, IgG(N, S) levels still surpassed this threshold at day 360 (Fig. 1E-F). In terms of gender, men showed higher levels than women at day 30 for all antibodies (Fig. 1G-I) and days 60 and 90 for IgG(S) (Fig. 1I).

\section{Note}

Antibody levels are represented with a boxplot together with a dot and text describing their median value. The dashed and the solid horizontal lines represent the uncertainty and positivity thresholds, respectively. Figures $1 \mathrm{~A}-\mathrm{C}$ show the significant differences in the median antibody levels between days. Figures 1D-F show significant differences in the median antibody levels across disease severity at each timepoint. Finally, Figs. 1G-I show the significant differences in antibody levels between genders at each timepoint. Significance levels were reported as: * for $p$-value $\leq 0.05$; ** for $p$-value $\leq 0.01$; *** for $p$-value $\leq$ 0.001 ; and $* * * *$ for $p$-value $\leq 0.0001$.

\section{Kinetics of IgM and IgG}

$\lg G(N)$ and $\lg G(S)$ had a different kinetics. In relation to the kinetics of the three antibodies stratified by disease severity, both LOESS (Figs. 2A, 2C, 2D) and NLME (Figs. 2B, 2D, 2E) showed a general rise in both $\operatorname{lgM}(\mathrm{N})$ and $\lg \mathrm{G}(\mathrm{N})$ levels up to day 30 followed by a decay, and both the height of the peak and the rate of decay depended on disease severity. IgG(S) levels increased at day 15 and remained relatively constant over time.

\section{Note}

Figures $2 \mathrm{~A}, 2 \mathrm{C}$ and $2 \mathrm{E}$ show the LOESS regression models, connecting datapoints belonging to the same participants. Figures 2B, 2D and 2F show the estimated non-linear mixed-effect (NLME) model curves. Each point corresponds to the mean value at each time point. The bars correspond to the standard deviation. The dashed and the solid horizontal lines represent the uncertainty and positivity thresholds, respectively.

Table S3 shows the parameters estimated for each component of the NLME curves. Q-Q plots of each model are available in Figure S2. For both $\lg \mathrm{M}(\mathrm{N})$ and $\lg \mathrm{G}(\mathrm{N})$, initial levels were significantly higher in severe-critical. $\lg \mathrm{M}(\mathrm{N})$ levels from asymptomatic participants decreased quicker, while those of severe-critical decreased slower than mild-moderate participants. This slower decline also occurs in $\lg G(N)$. Similarly to previous analyses, levels of $\lg G(S)$ increased with disease severity for all timepoints, and the levels were practically constant from day 30 in all groups. 
Figure 3. Kinetics of $\lg M(N), \lg G(N)$ and $\lg G(S)$ levels since diagnosis in the total sample and stratified by gender. Figures $3 A$, $3 \mathrm{C}$ and $3 \mathrm{E}$ show the LOESS regression models, connecting datapoints belonging to the same participants. Figures $3 \mathrm{~B}, 3 \mathrm{D}$ and 3F show the estimated non-linear mixed-effect (NLME) model curves. Each point corresponds to the mean value at each time point. The bars correspond to the standard deviation. The dashed and the solid horizontal lines represent the uncertainty and positivity thresholds, respectively.

\section{Discussion}

Our study draws strength from using longitudinal data from a large, representative, well characterized, and stratified sample of health professionals. The immune response to SARS-CoV-2 differs across disease severity. Our results show that antibody response starts within 15 days of the infection for the three studied isotypes. Thereafter, their behaviour diverges according to disease severity. We found a higher level of antibodies in patients with severe versus asymptomatic infections [11]. Our results also corroborate the early appearance of $\lg G(\mathrm{~N})$ and $(\mathrm{S})$ [6].[14]

Asymptomatic individuals maintain antibody levels above the threshold for $\lg M(N)$ up to six months, while antibodies for $\lg \mathrm{G}(\mathrm{N})$ and $(\mathrm{S})$ remain above the positivity threshold until day 270. The long-term presence of $\lg \mathrm{G}(\mathrm{N})$ antibodies and their protective efficacy, however, needs further research [15], [16]. Compared with asymptomatic cases, participants with mildmoderate COVID-19 presented higher IgG antibody levels, and their $\operatorname{lgM}(\mathrm{N})$ levels also remained above the threshold for the entire follow-up. These findings are similar to those reported at six months in a longitudinal study of $\operatorname{lgM}(\mathrm{S})$ and can be explained by the differentiation of B cells to IgM memory plasma cells that continue to produce the IgM isotype antibodies for at least a year [17]. Another recent study also found positive IgM levels for up to one year, although the isotypes $\lg M(S)$ rather than $\lg M(N)$. Severe-critical participants had higher antibody levels than the other groups in the first 30 days of follow-up and maintained the highest levels for all three isotypes throughout the entire follow-up period. This is consistent with the results of other studies conducted over a shorter period of time [18]' [9].

The LOESS models describe the trajectories of the antibody levels, while the NLME allows to compare the stratified trajectories while accounting for the non-linearity of the antibody level kinetics. Our study further elaborates the kinetics of the humoral immune response while appropriately accounting for its characteristics and influencing factors as justified by the model selection. Our results are better equipped to inform social distancing and (re)vaccination policies. Furthermore, our analyses can be easily adapted for estimating antibody levels measured in other units by applying the corresponding conversion factors. Likewise, they can be also employed to investigate the kinetics after vaccination, thus assessing the effectiveness of the vaccines in comparison with the immunisation due to infection and informing the criteria for revaccination.

To date, our study provides the most detailed and comparative description of the kinetics of $\lg \mathrm{G}(\mathrm{N})$ and $\lg \mathrm{G}(\mathrm{S})$ production after SARS-CoV-2 infection. $\lg \mathrm{G}(\mathrm{N})$ levels show a rapid rise followed by a decay with a slope depending on disease severity, and it stabilizes between day 270 and 360 . In contrast, IgG(S) kinetics shows a plateau from day 30 post-infection. This difference has also been reported in another study [19] and may be attributable to the differences between the $S$ and $N$ proteins of SARS-COV-2 in their molecular structure, their abundance in the viral particle, and their specific functions. While the former facilitates the entry of the virus into the host cell, the latter has a role in viral genomic packaging [20]. Monitoring of both IgG N and S antibodies can help identify stimulation of memory cells triggered by the two different antigens. Turner et al [17] analized the frequency of S-specific plasma cells in bone marrow of convalescent individuals, and observed that infection with SARS-CoV-2 is able to induce resting memory B cells and long-lived bone marrow plasma cells, supporting the existence of a robust humoral immune memory in infected individuals. Cohen $\mathrm{KW}$ et al. confirmed the former results and also analysed specific T-cells to SARS COV-2 [21]. Interestingly, they found that CD4 + T cell responses equally target several SARS-CoV-2 proteins, whereas CD8 + T cells preferentially target the N protein, highlighting the potential importance of including this antigen in future vaccines [21]. The maintenance of an $\mathrm{N}$ and $\mathrm{S} T$ and $\mathrm{B}$ immune responses may protect against possible reinfections and render (re)vaccination redundant. Further in-depth immunological studies will be necessary to address their role in protection from severe cases, including death and reinfection after both infection and vaccination. [22] 
Humoral immune response seems to have a very similar trajectory in men and women, although we found higher $\lg G(\mathrm{~N})$ or $\lg \mathrm{G}(\mathrm{S})$ levels in men at all timepoints. Other studies with an eight-month follow-up indicate that gender and disease severity is associated due to differences in immune memory to SARS-CoV-2. However, most of the heterogeneity in immune memory to SARS-CoV-2 is still unexplained, and further investigation on the role of cellular immunity is also needed, especially in vulnerable groups, (non-seroconvertors, people with immunodeficiencies, and autoimmune disorders) [2]' [23].

Anti-SARS-CoV2 antibodies were determined by ELISA techniques. The maximum levels of $\lg M(\mathrm{~N})$ and $\lg \mathrm{G}(\mathrm{N})$ antibodies tested could be even higher than reported, as the technique used was semi-quantitative due to the limited availability of quantitative in vitro diagnostic techniques at the beginning of the pandemic. Although, the WHO's international standard for quantification of $\operatorname{lgG}(\mathrm{S})$ antibodies was established after the study start, we retrospectively analysed the samples for $\lg \mathrm{G}(\mathrm{S})$ using a quantitative technique. The follow-up of $\lg \mathrm{G}(\mathrm{S})$ levels stopped as participants were vaccinated, while the $\operatorname{lgM}(\mathrm{N})$ and $\lg \mathrm{G}(\mathrm{N})$ continued.

Interpretation of these results should consider the limitations of the study. Frist, the majority of the ProHEpIC-19 sample are of white European origin, so findings might not be generalizable to other ethnic groups.

As more and more people are getting vaccinated, it is important to understand whether and how the immunity due to vaccination is different from that due to the infection. Are the kinetics and the durations of the antibody levels similar? Epidemiological modelling studies, especially long-term immunity monitoring, are focusing on the SARS-CoV-2 nowadays, however, one must also evaluate the interactions between SARS-CoV-2 and other coronaviruses for potential crossimmunisation [22][24]. Moreover, epidemiological modelling of SARS-CoV-2 can also benefit from that of other viruses, such as flu and HIV-1; and vice versa [25]' [26].

The code to specify and fit the NLME is available, and one can exploit and extend as they see fit, and the results of this study will inform the clinical practice guidelines to assess the SARS-COV-2 seroprevalence.

To conclude, we monitored three types of antibodies for 17 months, and analysed their levels and kinetics while also considering the effect of gender and disease severity. NLME models allowed a more detailed understanding of the trajectories, confirming that infected people can maintain immunity due to the prolonged seroprevalence of IgG isotypes. While our study focused on the health care workers due to their importance in fighting against the pandemic, larger studies with a more heterogeneous sample and longer follow-up period can provide better generalisability and potentially elaborate on further effects. Even though the fitted estimating equations for the evolution of each antibody levels are provided in the Supplementary Material, NMLE models should be considered as a method to study the temporal evolution, instead of a punctual estimator. Due to the standardised quantitative assessment of $\lg \mathrm{G}(\mathrm{S})$, our kinetic models can serve as a reference for future studies and inform social distancing and vaccination strategies.

\section{Declarations}

\section{Declaration of interests}

All authors have completed the ICMJE uniform disclosure form at www.icmje.org/coi_disclosure.pdf and declare no support from any organization for the submitted work other than that detailed in the Funding section. All other authors declare no competing interests.

\section{Data sharing}

No patient level data can be shared in accordance with the local information governance and data protection regulations. The code used for the statistical analysis is available on GitHub (https://github.com/IDIAPJGol/ProHEpic_Antibodies). We have made available several tables generated with synthetic data that can be used to run the scripts. The code and synthetic data 
are available and reported in the supplementary material immediately following publication and ending on May 31, 2035. Protocol and informed consent are published on ClinicalTrials.gov (NCT04885478).

\section{Acknowledgments}

The authors would like to sincerely thank the participants for their effort and selfless involvement in the ProHEpiC-19 cohort study. Also, they want to thank the Management Department, Primary Care Directorate, and the Directorate of the Clinical Laboratory of the Metropolitan North for the facilities they have given for the project. In addition, we deeply thank the technical staff of IrsiCaixa for processing the samples. We thank "CERCA Programme/Generalitat de Catalunya" for institutional support.

\section{Funding}

This project has been funding by the regional Ministry of Health of the Generalitat de Catalunya (Call COVID19-PoC SLT16_04) the Carlos III Health Institute (Ministry of Economy and Competitiveness, Spain) through the RETICS RD16/0007/0001 and RETIC RD16/0025/0041), the European Union European Regional Development Fund (ERDF) funds, the Catalan Government (Grant Number AGAUR 2017 SGR 445), and thePI19 Contratos predoctorales de formación en investigación en salud (AES 2019).

\section{Disclosure of results before publication}

A previous version of this work has been published in MedRxiv

https://www.medrxiv.org/content/10.1101/2021.09.10.21262527v1

\section{References}

1. Galit and Seder RGA. The Power of Antibody-Based Surveillance. N Engl J Med 2020;383:1782-4. https://doi.org/10.1056/nejme2029532.

2. Dan JM, Mateus J, Kato Y, Hastie KM, Yu ED, Faliti CE, et al. Immunological memory to SARS-CoV-2 assessed for up to 8 months after infection. Science (80-) 2021;371. https://doi.org/10.1126/science.abf4063.

3. Trinité B, Tarrés-Freixas F, Rodon J, Pradenas E, Urrea V, Marfil S, et al. SARS-CoV-2 infection elicits a rapid neutralizing antibody response that correlates with disease severity. Sci Rep 2021;11. https://doi.org/10.1038/s41598-021-81862-9.

4. Carrillo J, Izquierdo-Useros N, Ávila-Nieto C, Pradenas E, Clotet B, Blanco J. Humoral immune responses and neutralizing antibodies against SARS-CoV-2; implications in pathogenesis and protective immunity. Biochem Biophys Res Commun 2021;538:187-91. https://doi.org/10.1016/j.bbrc.2020.10.108.

5. Wang P, Liu L, Nair MS, Yin MT, Luo Y, Wang Q, et al. SARS-CoV-2 neutralizing antibody responses are more robust in patients with severe disease. Emerg Microbes Infect 2020;9:2091-3. https://doi.org/10.1080/22221751.2020.1823890.

6. Long QX, Tang XJ, Shi QL, Li Q, Deng HJ, Yuan J, et al. Clinical and immunological assessment of asymptomatic SARSCoV-2 infections. Nat Med 2020;26:1200-4. https://doi.org/10.1038/s41591-020-0965-6.

7. Gudbjartsson DF, Norddahl GL, Melsted P, Gunnarsdottir K, Holm H, Eythorsson E, et al. Humoral Immune Response to SARS-CoV-2 in Iceland. N Engl J Med 2020;383:1724-34. https://doi.org/10.1056/nejmoa2026116.

8. Lumley SF, O'Donnell D, Stoesser NE, Matthews PC, Howarth A, Hatch SB, et al. Antibody Status and Incidence of SARSCoV-2 Infection in Health Care Workers. N Engl J Med 2021;384:533-40. https://doi.org/10.1056/nejmoa2034545.

9. Huang AT, Garcia-Carreras B, Hitchings MDT, Yang B, Katzelnick LC, Rattigan SM, et al. A systematic review of antibody mediated immunity to coronaviruses: kinetics, correlates of protection, and association with severity. Nat Commun 2020;11. https://doi.org/10.1038/s41467-020-18450-4.

10. Dobaño C, Ramírez-Morros A, Alonso S, Vidal-Alaball J, Ruiz-Olalla G, Vidal M, et al. Persistence and baseline determinants of seropositivity and reinfection rates in health care workers up to 12.5 months after COVID-19. BMC Med 
2021;19:155. https://doi.org/10.1186/s12916-021-02032-2.

11. Ortega N, Ribes M, Vidal M, Rubio R, Aguilar R, Williams S, et al. Seven-month kinetics of SARS-CoV-2 antibodies and role of pre-existing antibodies to human coronaviruses. Nat Commun 2021;12:1-10. https://doi.org/10.1038/s41467-02124979-9.

12. World Health Organization (WHO). Seroepidemiological investigation protocol for coronavirus 2019 (COVID-19) infection 2020;2020:https://www.who.int/publications/i/item/WHO-2019-n.

13. Mattiuzzo G, Bentley EM, Hassall M, Routley S. Establishment of the WHO International Standard and Reference Panel for anti-SARS-CoV-2 antibody. 2020.

14. Chen X, Chen Z, Azman AS, Deng X, Sun R, Zhao Z, et al. Serological evidence of human infection with SARS-CoV-2: a systematic review and meta-analysis. Lancet Glob Heal 2021;9:e598-609. https://doi.org/10.1016/S2214109X(21)00026-7.

15. Harrington WE, Trakhimets O, Andrade D V., Dambrauskas N, Raappana A, Jiang Y, et al. Rapid decline of neutralizing antibodies is associated with decay of IgM in adults recovered from mild COVID-19. Cell Reports Med 2021;2. https://doi.org/10.1016/j.xcrm.2021.100253.

16. Zhao J, Zhao S, Ou J, Zhang J, Lan W, Guan W, et al. COVID-19: Coronavirus Vaccine Development Updates. Front Immunol 2020;11:1-19. https://doi.org/10.3389/fimmu.2020.602256.

17. Turner JS, Kim W, Kalaidina E, Goss CW, Rauseo AM, Schmitz AJ, et al. SARS-CoV-2 infection induces long-lived bone marrow plasma cells in humans. Nature 2021. https://doi.org/10.1038/s41586-021-03647-4.

18. Graham NR, Whitaker AN, Strother CA, Miles AK, Grier D, McElvany BD, et al. Kinetics and isotype assessment of antibodies targeting the spike protein receptor-binding domain of severe acute respiratory syndrome-coronavirus- 2 in COVID-19 patients as a function of age, biological sex and disease severity. Clin Transl Immunol 2020;9.

https://doi.org/10.1002/cti2.1189.

19. Alfego D, Sullivan A, Poirier B, Williams J, Adcock D, Letovsky S. A population-based analysis of the longevity of SARSCoV-2 antibody seropositivity in the United States. EClinicalMedicine 2021;36:100902. https://doi.org/10.1016/j.eclinm.2021.100902.

20. Mariano G, Farthing RJ, Lale-Farjat SLM, Bergeron JRC. Structural Characterization of SARS-CoV-2: Where We Are, and Where We Need to Be. Front Mol Biosci 2020;7. https://doi.org/10.3389/fmolb.2020.605236.

21. Cohen KW, Linderman SL, Moodie Z, Czartoski J, Lai L, Mantus G, et al. Longitudinal analysis shows durable and broad immune memory after SARS-CoV-2 infection with persisting antibody responses and memory B and T cells. Cell Reports Med 2021;2:100354. https://doi.org/10.1016/j.xcrm.2021.100354.

22. Loyal L, Braun J, Henze L, Kruse B, Dingeldey M, Reimer U, et al. Cross-reactive CD4 + T cells enhance SARS-CoV-2 immune responses upon infection and vaccination. Science (80-) 2021;374. https://doi.org/10.1126/science.abh1823.

23. Kilpeläinen A, Jimenez-Moyano E, Blanch-Lombarte O, Ouchi D, Quirant-Sanchez B, Chamorro A, et al. Highly functional Cellular Immunity in SARS-CoV-2 Non-Seroconvertors is associated with immune protection 5. BioRxiv 2021:2021.05.04.438781.

24. Abela IA, Pasin C, Schwarzmüller M, Epp S, Sickmann ME, Schanz MM, et al. Multifactorial seroprofiling dissects the contribution of pre-existing human coronaviruses responses to SARS-CoV-2 immunity. Nat Commun 2021;12. https://doi.org/10.1038/s41467-021-27040-x.

25. Petersen E, Koopmans M, Go U, Hamer DH, Petrosillo N, Castelli F, et al. Comparing SARS-CoV-2 with SARS-CoV and influenza pandemics. Lancet Infect Dis 2020;20:e238-44.

26. Fischer W, Giorgi, Elena E Chakraborty, Srirupa Nguyen, Kien Bhattacharya, Tanmoy Theiler J, Goloboff PA, Yoon H, Abfalterer W, Foley BT, et al. HIV-1 and SARS-CoV-2: Patterns in the evolution of two pandemic pathogens. Cell Host Microbe 2021;29:1093-100. https://doi.org/10.1016/j.chom.2021.05.012.

\section{Figures}

Page 13/15 

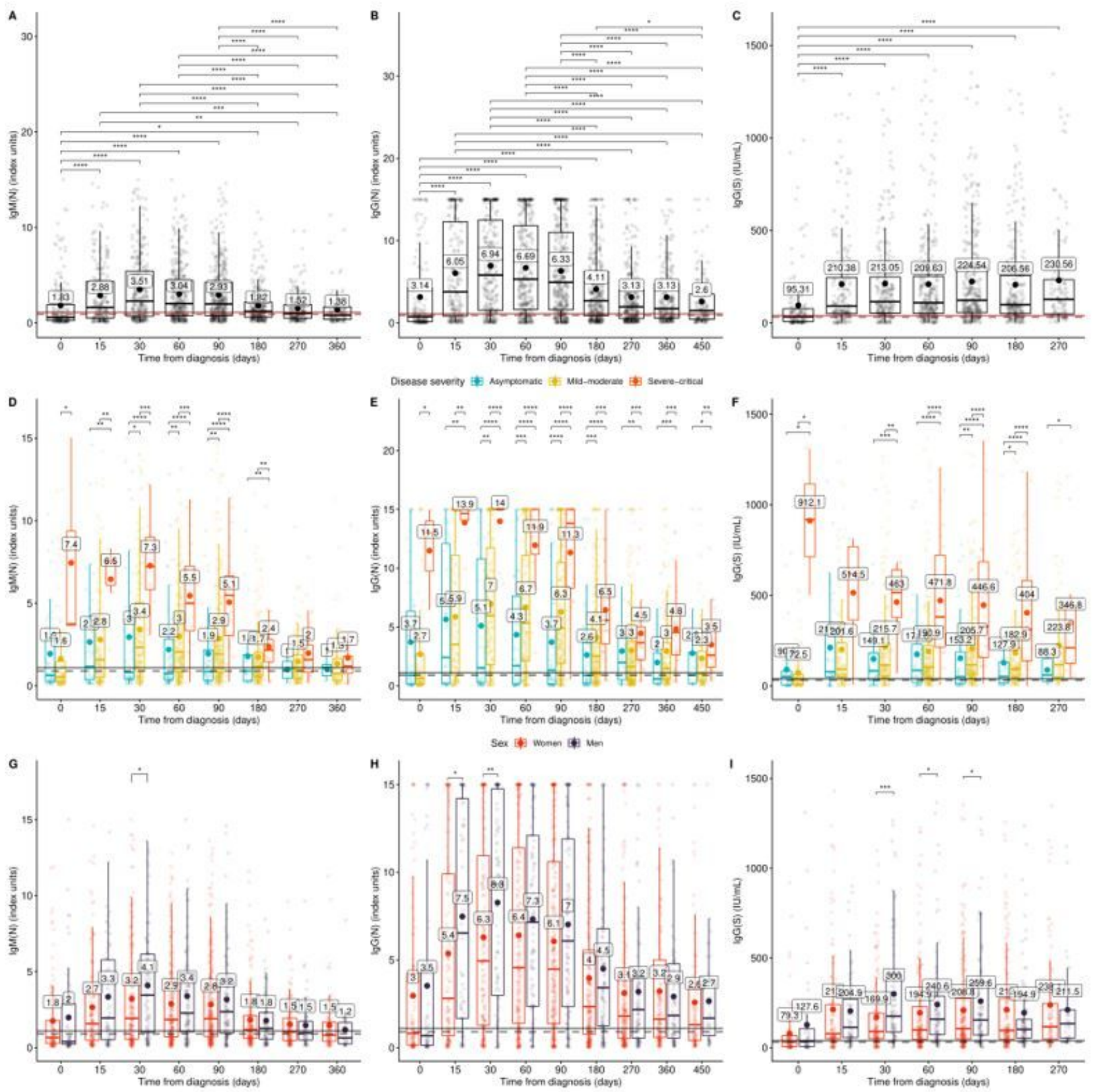

\section{Figure 1}

$\lg M(N), \lg G(N)$ ang $\lg G(S)$ levels, by days since diagnosis.

Note: Antibody levels are represented with a boxplot together with a dot and text describing their median value. The dashed and the solid horizontal lines represent the uncertainty and positivity thresholds, respectively. Figures 1A-C show the significant differences in the median antibody levels between days. Figures 1D-F show significant differences in the median antibody levels across disease severity at each timepoint. Finally, Figures 1G-I show the significant differences in antibody levels between genders at each timepoint. Significance levels were reported as: * for $p$-value $\leq 0.05$; ** for $p$-value $\leq 0.01$; *** for $p$-value $\leq 0.001$; and $* * * *$ for $p$-value $\leq 0.0001$. 

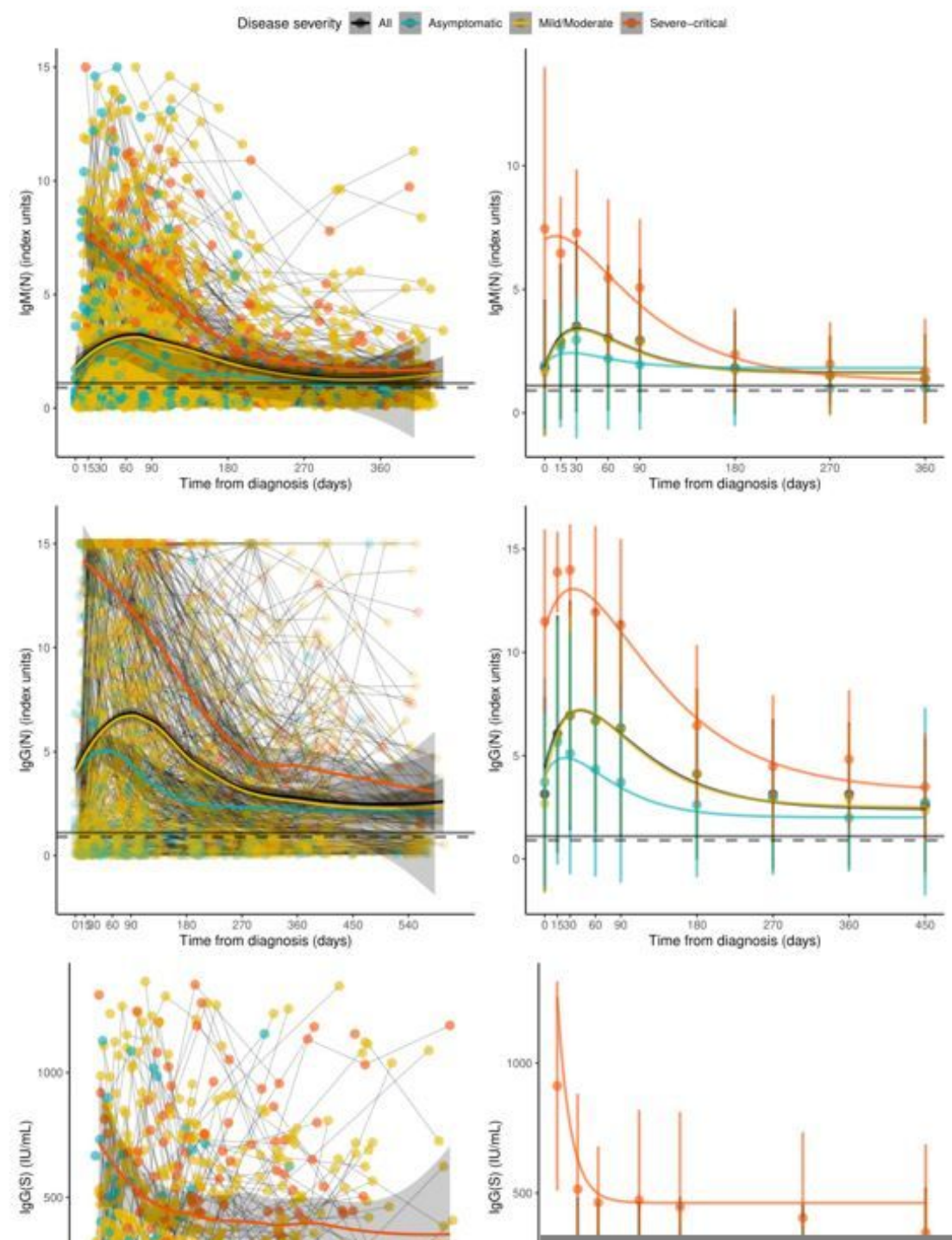

Figure 2

Kinetics of $\lg M(N), \lg G(N)$ and $\lg G(S)$ levels since diagnosis in the total sample and stratified by disease severity.

\section{Supplementary Files}

This is a list of supplementary files associated with this preprint. Click to download.

- FigS1.pdf

- Figs2.pdf

- SuplementaryProtocolDEF.pdf

- SupplementaryMaterialFinal.docx 